\title{
Mean shear resistance at steady-state for wet glass beads impact of liquid content and particle size
}

\author{
Haithem Louati, Driss Oulahna, and Alain de Ryck ${ }^{*}$ \\ Rapsodee Research centre, Mines Albi, CNRS University of Toulouse, Campus Jarlard, F-81013 Albi Cedex 09, France
}

\begin{abstract}
The flow behaviour of a granular media is due to their weight, frictional contact forces between them, and external forces exerted by the walls. If their size is lower than 50 microns, the Van-der-Waals forces between them may also influence their flowability. When adding some wetting liquid, we introduce attractive forces between the particles, whose order of magnitude may overcome the particle weight and V$\mathrm{d}-\mathrm{W}$ interactions. This leads to a cohesive behaviour. The shear stress to start the flow is greater than in the dry case but the steady-state flow is also perturbed by the presence of liquid bridges. This later phenomenon has been recently quantitatively studied for 70-110 $\mu \mathrm{m}$ glass beads with a non-volatile liquid, with experimental results for different normal stresses (up to $12 \mathrm{kPa}$ ) and liquid content (up to $20 \%$ in volume). These results have been compared to a heuristic model, based on the model for capillary bridges and the simplest hypothesis for the granular bed texture depending on the stresses applied. We extend this study with new results concerning smaller glass beads $12-40 \mu \mathrm{m}$ in diameter and larger liquid fraction for 70-110 $\mu \mathrm{m}$ glass beads using experimental and theoretical approaches.
\end{abstract}

\section{Introduction}

The introduction of a small amount of wetting liquid in a granular media leads to enhance its shear resistance if the particle size is typically lower than a few $\mathrm{mm}$ (the capillary forces exerted by the liquid menisci are then greater than the particle weight). This behaviour also occurs for a large liquid content till the full filling of the interparticle space. The strengthening is due firstly to the capillary pressure raising from the curvature of liquid-air interface imposed by the solid geometry and fluid incompressibility; and secondly to the liquid-air interface at the solid surface. Depending on the specific proportion of liquid, Newitt and Conway [1] defined the pendular, funicular and capillary regimes for separated liquid bridges, partially and fully filling of the space between particles. This behaviour has been studied by the observation of the limit of static configurations by Hornbaker et al. [2], Fraysse et al. [3] and Bocquet et al. [4]. To quantify more this effect in a dynamic situation, we have performed in a previous study by Louati et al. [5], a series of experiments where the shear resistance of $70-110 \mu \mathrm{m}$ glass beads, wetted by Polyethylene Glycol of $400 \mathrm{~g}$ molar weight, is measured in steady-state conditions for different normal loadings. Depending on both parameters (liquid fraction $\mathrm{V}_{\mathrm{R}}$ and normal stress $\sigma$ ), the apparent behaviour is either frictional (shear stress proportional to the normal stress) or presents an apparent cohesion (for normal stresses $\sigma>4 \mathrm{kPa}$ ). These two behaviours have been semi-quantitatively explained by

\footnotetext{
*Corresponding author: alain.deryck@mines-albi.fr
}

evaluating the number of capillary bridges renewed during shear at low and high normal stresses. These effects depend on the particle size. We present here how the size reduction affects the shear resistance in steadystate for partially wet granular media, with a liquid content up to the saturation of the interparticles space (60\% in our experiments).

\section{Materials and methods}

The glass beads used as model materials in this study are supplied from Sovitec ${ }^{\circledR}$ and sieved to obtain a narrow size distribution in the range of 70-110 $\mu \mathrm{m}$ and 12-40 $\mu \mathrm{m}$ in diameter. These sizes of glass beads are chosen relatively small to enhance the effect of the capillary forces over the gravitational forces. The surface of the material, analysed using SEM, shows small asperities in the range of 1 to $2 \mu \mathrm{m}$ regardless the size of glass beads. This information is needed for the calculation of the capillary forces according to Adams and Perchard Approach [6], for the minimal distance separation between the beads.

Polyethylene glycol 400 (PEG 400) from Alfa Aesar was used to prepare the wet glass beads samples. This liquid wets well the glass surface with an angle of contact close to $0^{\circ}$. The density of PEG 400 is $1.128 \mathrm{~g} / \mathrm{cm}^{3}$, and the surface tension is $56.74 \mathrm{mN} / \mathrm{m}$. The fraction of liquid is expressed as the volume of liquid relative to the volume of the glass beads sample, denoted by $\mathrm{V}_{\mathrm{R}} \%$. The fractions of liquid studied vary from $0.01 \%$ to $20 \%$ for the partially state of saturation for both sizes of glass 
beads. $\mathrm{V}_{\mathrm{R}}$ is then increased up to $60 \%$ for the $70-110 \mu \mathrm{m}$ glass beads to explore more saturation regimes.

In order to study the shear behaviour of the wet glass beads, a Schulze shear cell was used. This device consists of an annular shear cell of external and internal diameters of 10 and $20 \mathrm{~cm}$ respectively. The shear cell filled with the glass beads rotates at constant velocity of $2.3 \mathrm{mrad} / \mathrm{s}$, while the lid is maintained fixed. The lid has bars to avoid wall slippage and the shear plane is occurred at the level of these bars after rupture of the granular material (see Fig. 1). The shear test consists of measuring the shear stress $\tau$ under different normal stresses $\sigma$ applied to the granular material. We investigate the shear response at the steady-state situation. Further information about this device and the shear test procedure can be found in [5].

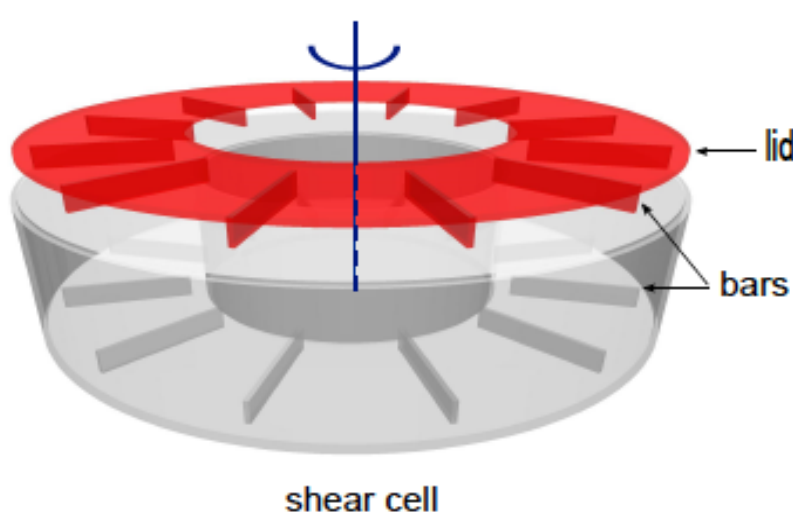

Fig. 1. Schema of the annular shear cell.

\section{Results and discussions}

\subsection{Impact of the particle size on the apparent friction of partially wet glass beads at low normal stress}

Figure 2 shows the apparent friction (the so-called bulk friction coefficient) calculated from the slope of the curves given by the shear stress as a function of the normal stress from the shear test experiments. The data are plotted in blue triangles for the 70-110 $\mu \mathrm{m}$ glass beads, and in green circles for the smaller beads (12-40 $\mu \mathrm{m})$. The data are obtained for low normal stress $\sigma$ less than $4 \mathrm{kPa}$ where the shear stress $\tau$ is proportional to $\sigma$ for both particle sizes, or linear with a small apparent cohesion (studied in section 3.2.1). The error bars indicate the incertitude of the measure. For the small glass beads, this frictional behaviour extends on the whole range of normal stresses applied in our experiments and the green circles represents therefore the shear behaviour up to $\sigma=12 \mathrm{kPa}$. This is not the case for the 70-110 $\mu \mathrm{m}$ glass beads, for which a slope change is observed in the $\tau-\sigma$ curve around $\sigma=4 \mathrm{kPa}$. Beyond that value, the $70-110 \mu \mathrm{m}$ glass beads present a larger apparent cohesion during steady-state shear, studied further in section 3.2. This difference between the $12-40$ and $70-110 \mu \mathrm{m}$ at higher normal stresses is detailed in [7].

From fig. 2, we observe a similar behaviour of the apparent friction for an increase of the liquid fraction for both sizes: a quick increase of the apparent friction from 0.4 to around 0.6 for a small increase of the liquid fraction (less than $5 \%$ ) and then a saturation around 0.65 . This assumes that the liquid bridges formed between grains contribute to the increase of the apparent friction of the granular material during shearing: in addition to the grain-grain friction, the rupture of the liquid bridge is necessary to provoke the shearing.

At low liquid filling, the liquid bridge strength is mainly independent on the particle diameter but induced by the surface roughness size since the latter fixes the curvature of the liquid bridge. Since the surface asperities are similar in size around one $\mu \mathrm{m}$ for both particle sizes, it is expected a similar apparent friction. This low content stands for quite low liquid content $(0.1 \%$ or $0.01 \%$ for respectively the small and larger beads). For larger liquid content, the capillary bridges have a curvature fixed by the particle diameter and the capillary force remains mainly constant, giving this saturation or a liquid content from 5 to $20 \%$. Finally, the independency of the saturation on the particle size indicates that during shearing at low normal stresses, the weakest capillary bridges are broken and/or the others bonds partially mobilized. This phenomenon needs to be further studied in order to identify the mechanisms. As yet mentioned, this independency of the shear behaviour on the particle size does not occur at high normal stresses, and discussed in the section 3.2.

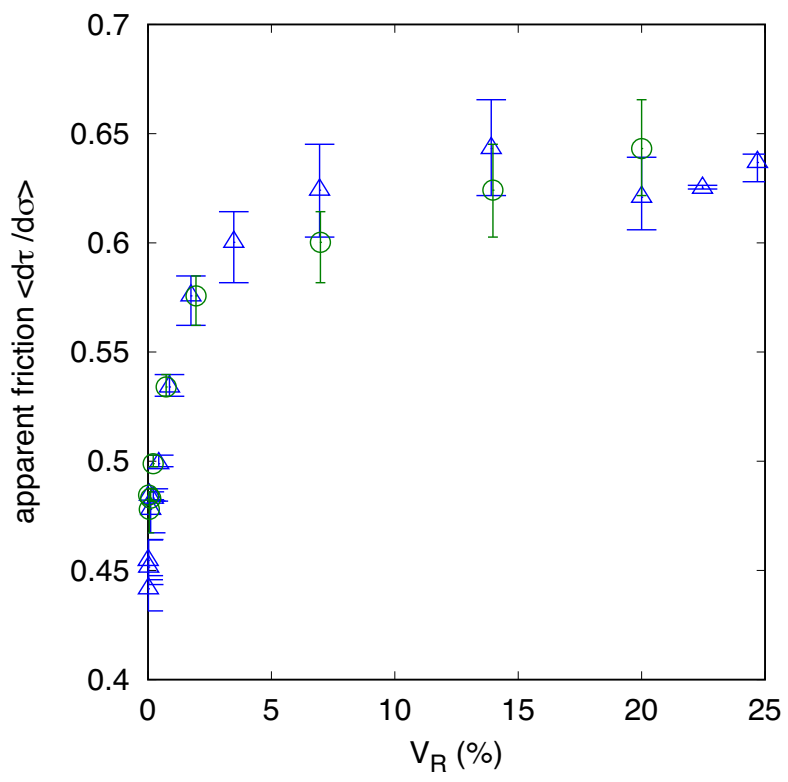

Fig. 2. Variation of the apparent friction at low normal stresses $(\sigma<4 \mathrm{kPa})$ with the liquid fraction for two sizes of glass beads (blue triangles: $12-40 \mu \mathrm{m}$ glass beads; green circles: 70-110 $\mu \mathrm{m})$. 


\subsection{Investigation of the tensile strength of wet glass beads for different saturation states}

For the $70-110 \mu \mathrm{m}$ glass beads, the frictional behaviour ends for a normal stress around $4 \mathrm{kPa}$ and the shear stress $\tau$-normal stress relationship at higher $\sigma$ is best fitted by:

$$
\tau=\mu\left(\sigma+T_{S}\right)
$$

where $\mu$ is an apparent friction close to the one observed for dry beads and $T_{S}$ is an apparent tensile strength dependent on the liquid content $\mathrm{V}_{\mathrm{R}}$. In order to investigate this tensile strength, experimental and theoretical approaches have been considered. Theoretically, the tensile strength is estimated using Rumpf model [4] for mono-sized spherical particle in the pendular regime (partially saturation of liquid), which states:

$$
T_{S, p}=\frac{1-\varepsilon}{\pi} \kappa \frac{F_{C}}{d^{2}}
$$

where $T_{S, p}$ is the tensile strength in the pendular regime, $\varepsilon$ is the void fraction of the wet glass beads, $\kappa$ is the coordination number, $d$ is the particle diameter and $F_{C}$ is the capillary force. The latter is calculated using liquid bridge model, as described by Adams and Perchard [1, 2].
In the capillary regime, where the liquid fills all the gaps between grains, the capillary force act through the capillary pressure at the agglomerate surface. Schubert proposed a model to estimate this tensile strength $[9,10]$ :

$$
T_{S, c}=a^{\prime} \frac{1-\varepsilon}{\pi} \kappa \frac{\gamma}{d} S
$$

where $T_{S, c}$ is the tensile strength in the capillary regime, $a^{\prime}$ is a dimensionless factor ranging from 6 and 8 depending on the particle rearrangement and $S$ is the liquid saturation given as the volume of liquid relative to the volume of void in the glass beads. In the funicular regime, it has been reported by Pierrat and Caram [10] that zones with separated liquid bridges and zones with glass beads totally saturated of liquid coexist. Therefore the capillary force arises from the liquid bridges together with the capillary pressure. The tensile strength is given as:

$$
T_{S, f}=T_{S, c} \frac{S_{2}-S}{S_{2}-S_{1}}-T_{S, p} \frac{S-S_{1}}{S_{2}-S_{1}}
$$

where $S_{1}$ is the limit saturation between the pendular and the funicular regimes and $S_{2}$ is the limit saturation between the funicular and the capillary regimes.

The Tensile strength has been investigated in our previous work [1]. We have distinguished between two regimes depending on the applied normal stress to the wet glass beads.

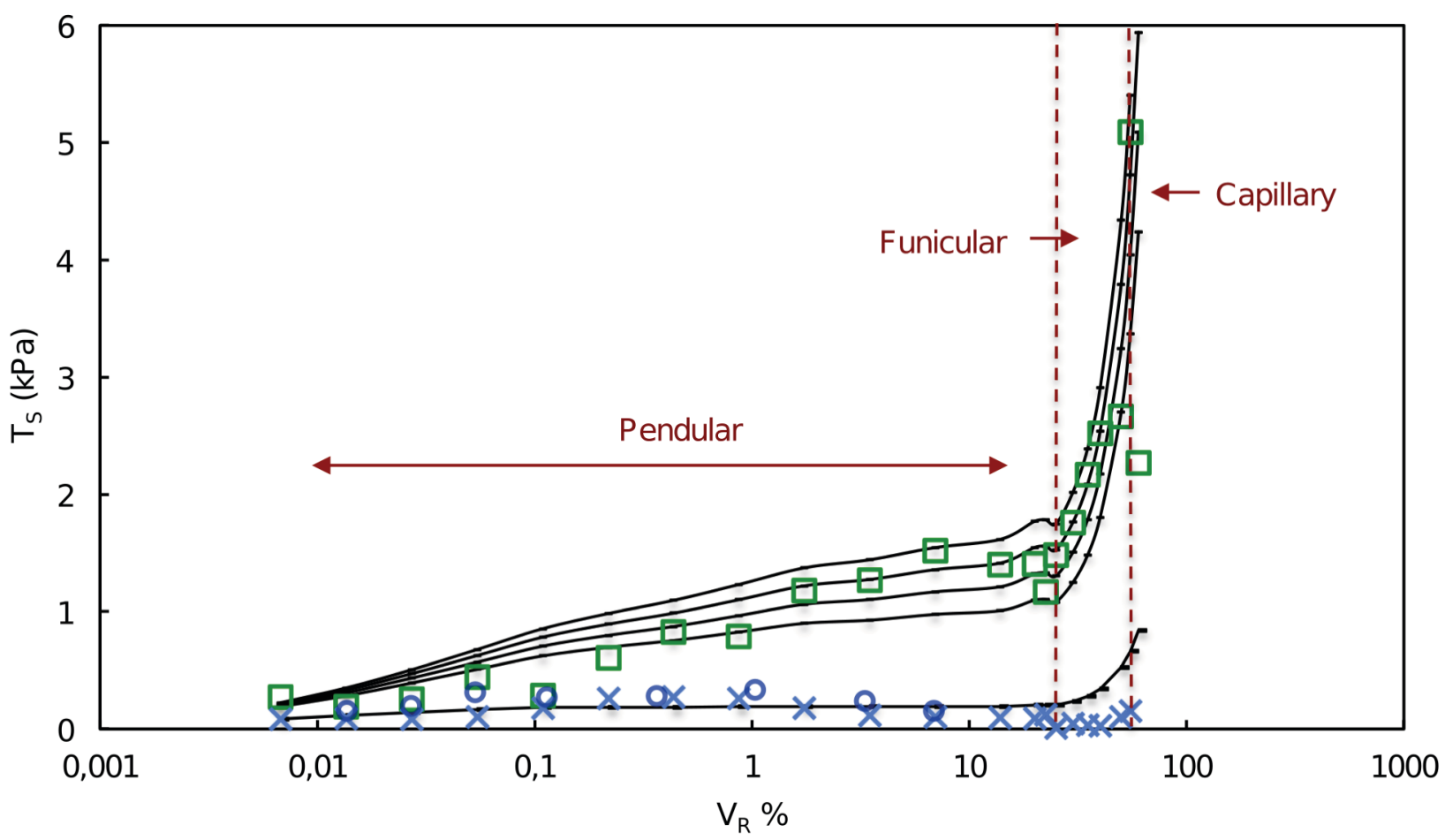

Fig. 3. Experimental and theoretical tensile strength as a function of the liquid fraction on a semi-logarithmic scale in the 70-110 $\mu \mathrm{m}$ case. Green squares and blue crosses: experimental data for the 70-110 $\mu \mathrm{m}$ glass beads at high normal stresses $(\boldsymbol{\sigma}>\mathbf{4} \mathbf{k P a})$ and low normal stresses $(\boldsymbol{\sigma}<\mathbf{4} \mathbf{~ k P a})$ respectively. Blue circles: experimental data for the $12-40 \mu \mathrm{m}$ glass beads. Continuous lines: theoretical tensile strength for different coordination numbers $(1,5,6,7$ and 8$)$ shown from bottom to top. 


\subsubsection{Low normal stress regime for both sizes}

At low normal stress $(\sigma<4 \mathrm{kPa})$ for the largest beads or for the whole range of normal stresses for the smallest beads, the tensile strength remains low (see blue crosses and circles respectively in Fig. 3) due to a loose configuration of the granular material, inducing a small number of liquid bridges broken explained by rolling rearrangements of glass beads. This small residual tensile strength or apparent cohesion is of order the one we compute with the hypothesis of one capillary bridge per particle broken in the shear plane (lowest continuous line in Fig. 3).

\subsubsection{High normal stress regime for largest size}

At high normal stress $(\sigma>4 \mathrm{kPa})$, the tensile strength increases more significantly with the liquid fraction compared to the one at low normal stress (green squares in Fig. 3). This is explained by the increase of the number of liquid bridges broken in the shear plane when the wet glass beads bed occurred a dense configuration due to the applied normal stress. In the range labelled Pendular, the square data are compared with 4 continuous lines obtained from Eq. 1 with a coordination number variation from 5 to 8 . The lines are not perfectly smooth because we have used the experimentally measured values for the void fraction $\varepsilon$. (We remind that this regime is not attained for the smallest particles).

In the continuation of these observations, we investigate the tensile strength for other saturation regimes implying eqs. 1, 2 and 3. Figure 3 shows a regime change of the tensile strength variation in the funicular regime. This appears in the graph as a remarkable increase of the tensile strength at high applied normal stress (squares). We assume that the normal stress applied to the wet glass beads affects their packing density. Indeed, agglomerates may be formed in the funicular regime. Thus, at low normal stress, the tensile strength required to separate agglomerates is lower compared to the case of packed granular bed at high normal stress. Moreover, the rolling rearrangement may be enhanced in the presence of agglomerates at low normal stress. This analysis based on the applied normal stress calls attention to the granular bed texture, which affects largely the wet granular materials.

The transition from the funicular to the capillary regime is difficult to identify. The tensile strength supposes to increase further in the capillary regime and vanish after that indicating the slurry regime $[11,12]$. Theoretically, the capillary regime is not supposed to be reached for liquid fraction up to $60 \%$. However, we observe a decrease of the tensile strength for the experimental point at high normal stress. This means that under high applied normal stresses, the void fraction in the granular bed is reduced and consequently the saturation of liquid is modified.

\section{Conclusions and perspectives}

In this study we have been focusing on the effect of the particle size and the liquid content on the shear behaviour of wet glass beads. It was observed that decreasing the size of particles leads to increase the shear resistance of the wet granular material due to the increasing number of broken liquid bridges in the shearing plane. The liquid bridges help increase the apparent friction. Thus, a saturation regime of the apparent friction was observed for the large glass beads after adding a small amount of liquid. While, the apparent friction continues to increase for the small glass beads since the saturation in the number of liquid bridge is not reached yet.

The effect of the liquid content was investigated by looking at the tensile strength variation with the liquid fraction. It was observed a regime change from the pendular to the funicular states of saturation. This is appeared as a remarkable increase of the tensile strength at high normal stress in the funicular regime. However, this tensile strength decreases at low normal stress, which highlights the effect of packing on the behaviour of wet granular materials. The packing of the wet glass beads was investigated using X-ray tomography analysis and has been submitted for publication [7].

\section{References}

1. D.Newitt,J.Conway-Jones, Trans. Inst. Chem. Eng. 36, 422-442 (1958).

2. D. Hornbaker, R. Albert, I. Albert, A. Barabasi, P. Schiffer, Nature 387, 765 (1997).

3. N. Fraysse, H. Thomé, L. Petit, Eur. Phys. J. B 11, 615-619 (1999).

4. L. Bocquet, É. Charlaix, F. Restagno, C. R. Phys. 3, 207-215 (2002).

5. H. Louati, D. Oulahna, A. de Ryck, Powder Technol. 278, 65-71 (2015)

6. M. Adams, V. Perchard, Inst. Chem. Eng. Symp. 91, 147-160 (1985)

7. H. Louati, D. Oulahna, A. de Ryck, submitted to Powder Technol.

8. H. Rumpf, Intersci. Publ. 379-418 (1962)

9. H. Schubert, Powder Technol. 37, 105-116 (1984)

10. P. Pierrat, H.S. Caram, Powder Technol. 91, 83-93 (1997)

11. N. Mitarai, F. Nori, Adv. Phys. 55, 1-45 (2006)

12. W. Pietsch, Agglomeration process (Wiley-VCH, Weinhein, 2002) 